\title{
An Excised-Leaf Inoculation Technique for Evaluating Host-Pathogen Interactions and Quantitative Resistance of Bermudagrass Genotypes to Dematiaceous Hyphomycetes
}

\author{
R. G. Pratt
}

U.S. Department of Agriculture-Agricultural Research Service, Waste Management \& Forage Research Unit, Mississippi State, MS 39762. Accepted for publication 7 August 2003.

\section{ABSTRACT}

Pratt, R. G. 2003. An excised-leaf inoculation technique for evaluating host-pathogen interactions and quantitative resistance of bermudagrass genotypes to dematiaceous hyphomycetes. Phytopathology 93:15651571.

Excised leaves of bermudagrass were inoculated with mycelium of isolates of Bipolaris, Exserohilum, Curvularia, and Drechslera spp. in water agar plates to evaluate differences in susceptibility of leaf tissue, virulence of pathogens, and quantitative resistance of bermudagrass genotypes. Isolates of nine species of pathogens induced similar symptoms of light- to dark-brown necrosis and bordering chlorosis in excised leaves that were not distinct for individual species or genera. Severity of symptoms induced by most isolates increased progressively from younger to older leaves. Within and across leaf positions, numerous significant differences in virulence of isolates within fungal species and between species were observed. Among 40 randomly selected bermudagrass genotypes, a continuous quantitative gradient was observed for mean scores of disease severity in excised leaves inoculated with $E$. rostratum. Numerous significant differences were observed within this gradient, and severity of symptoms in the most susceptible genotypes was approximately double that in the most resistant. When intact foliage of genotypes from the resistant and susceptible extremes of the gradient was inoculated with spores of E. rostratum, corresponding differences in severity of symptoms and significant $(P=0.05)$ correlations between results with excised leaves and intact foliage were observed. However, the range of differences in disease severity between genotypes was more narrow in intact foliage than in excised leaves. Results indicate that the excised leaf inoculation technique can be used to evaluate the relative resistance of bermudagrass genotypes to E. rostratum for use in programs to breed for quantitative host resistance.
Bermudagrass (Cynodon dactylon (L.) Pers.) is a perennial warm-season grass that is grown widely for both forage and turf in the southeastern United States (4). It also is the species most commonly grown on animal waste application sites to assimilate and enable removal of excess $\mathrm{P}$ from confined poultry and swine feeding operations $(14,15)$. Bermudagrass grows primarily from April through September or October in most areas of the Southeast and is dormant during winter months. During its period of active growth, bermudagrass may be damaged by a wide range of fungal diseases $(5,19,21)$.

Diseases caused by "dematiaceous hyphomycetes" (primarily species of Exserohilum, Bipolaris, Curvularia, and Drechslera) are some of the most important limiting factors for forage production, vigor, and persistence of bermudagrass and other warmand cool-season grasses in the southeastern United States $(5,6$, 14,19). Many pathogens of this group cause foliar leafspots or blights from which infection progresses basipetally into subterranean tissues to cause rotting and death of stems, stolons, rhizomes, and roots. Subterranean infection by dematiaceous hyphomycetes may result in stunting, loss of vigor, dieback, and death of portions of stands $(5,19)$.

On bermudagrass, Bipolaris cynodontis (Marignoni) Shoemaker, the causal agent of leaf blotch and stand dieback, often is considered the most common and important pathogen $(5,19,21)$. However, recently, many other species of dematiaceous hyphomycetes also were found to infect forage bermudagrass on animal

Corresponding author: R. G. Pratt; E-mail address: rpratt@msa-msstate.ars.usda.gov

Publication no. P-2003-1014-02R

This article is in the public domain and not copyrightable. It may be freely reprinted with customary crediting of the source. The American Phytopathological Society, 2003. waste application sites in Mississippi and to induce similar symptoms following spore inoculations $(14,15)$. Exserohilum rostratum (Drechs.) K. J. Leonard \& E. G. Suggs and B. spicifera (Banier) Subramanian were considered to be the major pathogens associated with severe stunting and dieback of stands on these sites (14).

Breeding for disease resistance appears to offer the best means for controlling diseases caused by dematiaceous hyphomycetes in bermudagrass grown for forage or turf. Among turfgrass cultivars, several are described as having high levels of resistance to $B$. cynodontis (6). Among cultivars of bermudagrass grown for forage, however, little breeding for resistance to dematiaceous hyphomycetes has been accomplished, and only cv. Coastal is reported to possess resistance to one species (possibly B. cynodontis) (4).

Development of genetic control for diseases caused by dematiaceous hyphomycetes in bermudagrass likely will require effective breeding for quantitative host resistance. Quantitative resistance refers to a system in which resistant phenotypes are conferred primarily by additive effects of few to numerous minor genes in favorable combinations, and differences in relative resistance and susceptibility of individuals within a population represent a continuous numerical gradient $(2,3,7)$. Effective breeding for quantitative resistance changes the mean of a population distribution by increasing the frequencies of favorable genes and gene combinations within a population while retaining sufficient genetic variation to avoid inbreeding depression and to enable further response to selection $(2,3,7)$. Although genes for quantitative resistance do not provide complete disease control, they are likely to be present in most populations, durable, effective against multiple races of pathogens if these exist, and sometimes effective against different genera of pathogens for which modes of parasitism are similar $(3,7,16)$. In contrast, breeding for qualitative resistance requires populations or individuals with highly resistant phenotypes that are conferred by dominant or recessive genes $(2,3,7)$, and this 
form of resistance is not known for dematiaceous hyphomycetous diseases in forage bermudagrass. In addition, species of Exserohilum and Bipolaris have demonstrated a strong potential for developing host-specialized pathogenic races that have rendered qualitative resistance genes ineffective in other hosts $(10,22)$.

A common practical requirement for effective breeding for quantitative resistance is that repeated tests must be performed to identify the most relatively resistant genotypes within a population, and to compensate for potentially strong environmental influences on the expression of resistance $(1,3,7,13)$. Spore inoculations of whole plants, which usually are very effective for screening large numbers of plants for qualitative resistance (11), may not be suitable for detecting smaller and more subtle differences in responses of individual genotypes that occur in quantitative systems (13). Excised leaf inoculation techniques, in contrast, offer excellent prospects for use in evaluating quantitative host resistance because large numbers of leaves can be obtained for assays, and repeated measurements of symptom severity in excised leaves enable discernment of small and subtle differences in host response and identification of variation caused by environmental influences. These techniques, if effective, also offer great improvements in efficiency of data collection because numerous tests can be repeated over time without extensive vegetative cloning of screened genotypes as would be required for conventional spore inoculations. Excised-leaf inoculation techniques have been used with a wide range of host-pathogen systems to evaluate differences in virulence of pathogens and resistance of hosts $(13,17)$. In one previous study with alfalfa, selections made only on the basis of excised leaf tissues in petri dishes enabled development of populations with effective resistance to natural disease caused by Sclerotinia trifoliorum Eriks. and increased yields in the field (13).

This study was undertaken to evaluate symptom development in excised bermudagrass leaves from different positions when inoculated with an array of dematiaceous hyphomycetous pathogens, and to determine whether excised leaf inoculations can be used to identify differences in quantitative resistance to E. rostratum among individual bermudagrass genotypes.

\section{MATERIALS AND METHODS}

Source, identity, storage, and growth of fungal isolates. Species of Bipolaris, Exserohilum, Curvularia, and Drechslera used in this study were isolated from symptomatic bermudagrass leaves, stems, and rhizomes collected on swine and poultry waste application sites in Mississippi (14,15), and isolates were identified by features of conidium and conidiophore morphology (5, $18,19)$. The only exceptions were two isolates of Bipolaris stenospila (Drechs.) Shoemaker (isolates 2 and 3) obtained from broadleaf signal grass (Brachiaria platyphylla (Griseb.) Nash). Identification of isolates of B. australiensis (M. B. Ellis) Tsuda \& Ueyanma from bermudagrass rhizomes is considered tentative because this species has not been reported previously in North America. Isolates of Drechslera sp. produced small spores that did not correspond clearly to those of described species $(5,18,21)$. Most isolates were maintained in dried, infested wheat and oat grain at $10^{\circ} \mathrm{C}$. Particles of infested grain were plated on Difco cornmeal agar (CMA) to initiate mycelial colonies. Isolates of $B$. australiensis, which did not survive on infested grain, were maintained as mycelial colonies at $5^{\circ} \mathrm{C}$.

Collection, inoculation, and evaluation of excised bermudagrass leaves. For experiments on effects of leaf position and fungal isolate on symptom development in excised leaves, leaves were collected from three positions on 108 growing stems selected randomly from among 200 genotypes (plants from individual germinated seed) of commercially available common bermudagrass (cultivar not designated; Oktibbeha County Co-Op, Starkville, MS) grown in a commercial enriched potting mixture
(Miracle-Gro Lawn Products, Marysville, $\mathrm{OH}$ ) in the greenhouse at 18 to $30^{\circ} \mathrm{C}$. Leaf positions 1,2 , and 3 were defined as the first, second, and third fully expanded leaves, respectively, proximal from a growing stem tip. A leaf was considered to be fully expanded when the blade was differentiated from the sheath and bent outward from the axis of the stem at $\geq 45^{\circ}$. Leaf sections extending from the base of the blade to $4 \mathrm{~cm}$ distal from the base were excised from leaves on growing stems. Nine sections of leaf tissue from the different positions were arranged radially at equidistant intervals on water agar (WA; $20 \mathrm{~g} / \mathrm{liter}, 50 \mathrm{ml}$ per plate) in 13.5-cm-diameter plates with adaxial surfaces facing upward, bases positioned near the centers, and distal cut ends extending to $1 \mathrm{~cm}$ from edges of plates, at positions preassigned by randomization of treatments. Leaf sections were inoculated by placing disks of agar (4 $\mathrm{mm}$ in diameter) over distal cut ends so that each disk overlapped halfway onto the leaf blade and halfway onto the agar in the plate. Agar disks were cut with a cork borer from margins of colonies grown on CMA. Plates were incubated under fluorescent plant growth lights (12) on a 12-h photoperiod at $26^{\circ} \mathrm{C}$ for 5 days. The length of continuous necrosis that extended proximally from the inoculated cut tip to the most distal healthyappearing, green tissue then was measured (in millimeters) under a dissecting microscope.

For experiments to compare responses in excised leaves of 40 additional, randomly selected bermudagrass genotypes to inoculation with $E$. rostratum, the same procedures were followed except that leaves were collected only from position 2 on stems, and 10 leaf sections were placed on each plate.

Inoculation and evaluation of intact foliage of plants. Of the 40 bermudagrass genotypes, 3 were selected for relative resistance and 4 for relative susceptibility to E. rostratum on the basis of excised-leaf inoculations; all 7 were vegetatively increased by rooting multiple stem cuttings in the commercial potting mixture in styrofoam cups (248-ml capacity). Plants were grown in the greenhouse, fertilized (30-30-30, N-P-K, soluble) at approximately 3-week intervals, and clipped back repeatedly to induce a thick growth of foliage. Cups of the seven genotypes with similar amounts of top growth were used in spore-inoculation experiments. Spores of E. rostratum were obtained by fragmenting $9 \mathrm{~g}$ (three isolates, $3 \mathrm{~g}$ each) of the dried, infested wheat and oat grain in a commercial food blender (Osterizer; Sunbeam Corp., Boca Raton, FL) for $10 \mathrm{~s}$. One gram of dry, comminuted particles was scattered over the surface of agar in a $13.5-\mathrm{cm}$-diameter plate of WA. After plates were incubated under growth lights for 10 days, spores were harvested by pouring $50 \mathrm{ml}$ of a dilute sticker solution (Pelgel, $1.0 \mathrm{~g} / 100 \mathrm{ml}$ of distilled water; Liphatech Corp., Milwaukee, WI) over the agar surface of each plate, scraping with a microspatula to dislodge the spores, and filtering the suspension through double-layer cheesecloth. Spore suspensions from several plates were combined to give approximately $600 \mathrm{ml}$ of suspension per experiment, with $6.8 \times 10^{3}$ spores $/ \mathrm{ml}$ as determined by four hemacytometer counts.

Cups containing the bermudagrass genotypes were placed on saturated paper towel within plastic rooting trays ( 52 by 26 by $6 \mathrm{~cm}$ ). Foliage of bermudagrass in all cups in each tray was sprayed uniformly to runoff with spore suspensions applied in a fine mist with a hand atomizer. Domed plastic covers were placed over trays to create saturated atmospheres, and plants were incubated for 3 days under the growth lights (12-h photoperiod) at $26^{\circ} \mathrm{C}$ to initiate infection and symptom development. Plants then were removed from trays, and the percentage of leaf and stem tissue that manifested known or potential disease symptoms (chlorosis, necrotic flecks, expanded individual necrotic lesions, lesions coalesced into streaks and zones of necrosis, and totally necrotic leaf blades and sheaths) was estimated visually for the total foliage in each cup. Plants then were returned to the greenhouse at 18 to $35^{\circ} \mathrm{C}$ for 7 additional days, at which time the total percentage of symptomatic tissue again was estimated for foliage in each cup. 
Experimental designs and statistical analyses. All experiments with excised leaf inoculations in petri plates, and spore inoculations of plants grown in cups, were established in randomized complete-block designs with four replicates. In experiments to evaluate responses of excised leaves from three positions on stems following inoculation with 26 fungal isolates plus controls (81 treatments), each block consisted of nine plates with nine leaves on each plate, among which leaf position and isolate combinations were completely randomized. The nine plates of each block were incubated together under plant growth lights following inoculation of leaves. The experiment was performed twice with four blocks, and results of each experiment were compared by analysis of variance (ANOVA) of a 3-by-27 factorial experiment. Means for significant $(P=0.05)$ main effects and for individual treatment combinations $(P=0.05)$ with significant interactions were compared by use of Fisher's protected least significant difference (LSD) test at $P=0.05$ (20).

In experiments to evaluate responses of excised leaves from 40 bermudagrass genotypes to $E$. rostratum following mycelial inoculation in plates, each block consisted of four plates with 10 leaves per plate, among which genotypes were completely randomized. The four plates of each block were incubated together following inoculation of leaves. The experiment was performed twice with four blocks, and results were combined for ANOVA after heterogeneity of variance and experiment-treatment interactions were declared nonsignificant at $P=0.05$. Treatment means were compared by use of Fisher's protected LSD test at $P=0.05$.

In experiments to compare severity of disease in seven bermudagrass genotypes following spore inoculations with $E$. rostratum, blocks consisted of single cups of all genotypes that were randomized together on a tray, sprayed simultaneously with spores of $E$. rostratum, and maintained together under growth lights and in the greenhouse following inoculation. Estimated percentages of leaves and stems that developed symptoms were compared by ANOVA following arcsin square root transformation of data (20). The experiment was performed twice with four blocks, and results were combined for ANOVA after heterogeneity of variance and experiment-treatment interactions were declared nonsignificant at $P=0.05$. Treatment means were compared by use of Fisher's protected LSD test at $P=0.05$.

\section{RESULTS}

Symptoms of infection in excised leaf tissues. Visible evidence of necrosis usually appeared beneath inoculum disks on cut leaf tips by $24 \mathrm{~h}$ after inoculation, and necrosis then spread basally into green leaf tissue. Most isolates induced a light- to mediumbrown necrosis with an irregular margin often preceded by chlorosis. Frequently, runner hyphae of a pathogen grew down the leaf surface ahead of the advancing margin of necrosis and initiated new, dark-brown lesions in green tissue that later were enveloped by spreading necrosis. Chlorosis and fading of green tissue ahead of margins of necrosis occurred most commonly in leaves from position 3 on stems. Symptoms induced by all species of dematiaceous hyphomycetes were indistinguishable; no individual species could be identified accurately on the basis of qualitative symptoms induced in excised leaf tissue.

Effects of leaf position and fungal isolates on severity of symptoms. In two experiments on effects of leaf positions and fungal isolates on symptom development in bermudagrass leaves, heterogeneity of variance was not significant, but significant experiment-treatment interactions were observed (Table 1). This indicated that effects of some treatments were inconsistent between experiments. Therefore, results were analyzed separately for each experiment.

In both experiments, significant variation was attributed to fungal isolates, leaf positions, and interactions between these factors.
On the basis of magnitude of $F$ values, variation attributed to leaf positions was greater in experiment 1 than in experiment 2 , and interactions between isolates and leaf positions were significant but minor compared with main effects in both experiments (Table 1).

Because of significant variation attributed to both main effects and interactions between treatments, significant differences are identified for main effects of fungal isolates across leaf positions (isolate means), main effects of leaf positions across isolates (leaf means), and individual effects of fungal isolates within leaf positions and leaf positions within isolates (Tables 2 and 3 ).

Across fungal isolates, severity of symptoms increased in leaf tissues from positions 1 to 3 in both experiments. In experiment 1 , severity of symptoms in leaf tissue from position 2 were intermediate and differed significantly from the other two positions; whereas, in experiment 2, results for leaf position 2 differed from those of position 3 but not position 1 (Tables 2 and 3). Within fungal isolates in experiment 1 , whenever significant differences in severity of symptoms between leaf positions were observed, symptoms were more severe in leaf tissue from position 3 than from position 1 . In experiment 2 , where fewer significant differences between leaf positions were observed (Table 3), for two isolates of $B$. stenospila, symptoms were more severe in leaf tissue from position 1 than from positions 2 or 3 (Table 3 ).

Across leaf positions, 24 and 21 isolates induced symptoms significantly more severe than in controls for experiments 1 and 2, respectively. Only isolates of $B$. australiensis and Drechslera spp. did not consistently induce symptoms more severe than in controls in both experiments; however, isolates of these two species grew at much slower rates than all others (data not shown). One isolate of B. stenospila from bermudagrass (isolate 1) was among the most virulent isolates in both experiments, whereas two isolates from broadleaf signal grass (isolates 2 and 3) were significantly less virulent (Tables 2 and 3). Among the remaining fungal pathogens, few consistent species-level differences in severity of symptoms were observed across leaf positions, and some individual isolates of most species were among the most virulent in one or both experiments. Differences within species in severity of symptoms usually were consistent across leaf positions, but some inconsistencies were observed (e.g., experiment 1, C. geniculata, position 1 versus 2 and 3 ; experiment 2, B. cynodontis, position 1 versus 3).

Responses of bermudagrass genotypes to excised leaf inoculations with $\boldsymbol{E}$. rostratum. The mean lengths of necrosis induced

TABLE 1. Sources of variation and their significance for experiments with isolates of dematiaceous hyphomycetous pathogens inoculated onto excised leaves of bermudagrass from positions on growing stems

\begin{tabular}{|c|c|c|c|}
\hline Experiment, source of variation ${ }^{\mathrm{z}}$ & $\mathrm{df}$ & MS & $F$ \\
\hline \multicolumn{4}{|l|}{ Combined experiments } \\
\hline Experiment & 1 & 1,599 & $101.67 * *$ \\
\hline Treatment & 80 & 191 & $12.02 * *$ \\
\hline Experiment-treatment & 80 & 27 & $1.69 * *$ \\
\hline Replicate & 3 & 419 & $26.19 * *$ \\
\hline Error & 486 & 16 & $\ldots$ \\
\hline \multicolumn{4}{|l|}{ Experiment 1} \\
\hline Isolate & 26 & 290 & $24.13 * *$ \\
\hline Leaf position & 2 & 933 & $77.75^{* *}$ \\
\hline Isolate-leaf position & 52 & 26 & $2.15 * *$ \\
\hline Replicate & 3 & 897 & $74.75^{* *}$ \\
\hline Error & 243 & 12 & $\ldots$ \\
\hline \multicolumn{4}{|l|}{ Experiment 2} \\
\hline Isolate & 26 & 215 & $16.54 * *$ \\
\hline Leaf position & 2 & 91 & $7.00 * *$ \\
\hline Isolate-leaf position & 52 & 17 & $1.32 *$ \\
\hline Replicate & 3 & 72 & $5.54 * *$ \\
\hline Error & 243 & 13 & $\ldots$ \\
\hline
\end{tabular}

$\mathrm{z}$ Isolate factor includes 26 fungal isolates plus one noninoculated control treatment. 
by an isolate of E. rostratum in excised leaves (position 2) from 40 randomly selected bermudagrass genotypes, in combined results of two experiments, are given in Figure 1. Means formed a continuous numerical gradient with numerous significant differences between individual genotypes. The lengths of necrosis induced in the most susceptible genotypes were approximately twice the lengths observed in the most resistant.

Responses of selected bermudagrass genotypes to spore inoculations with $\boldsymbol{E}$. rostratum. Mean percentages of symptomatic tissue observed in foliage of 7 of the 40 bermudagrass genotypes following spore inoculations with $E$. rostratum are presented in Table 4. Higher percentages of tissues were symptomatic at 3 days after inoculation than after 10 days, but significant differences between genotypes were observed after both intervals. Estimated percentages of tissue with symptoms of infection by $E$. rostratum were significantly less for three genotypes selected for resistance by excised leaf inoculations than in three of four genotypes selected for susceptibility at both evaluation times. Correlation coefficients for mean levels of necrosis in excised leaves and mean severity of symptoms in intact foliage of the seven bermudagrass genotypes at 3 and 10 days after inoculation were $r=0.84$ and 0.92 , respectively. Both coefficients were significant at $P=0.05$.

\section{DISCUSSION}

Results of this study demonstrate that inoculation of excised leaf tissues of bermudagrass with dematiaceous hyphomycetous pathogens is effective for comparing the virulence of isolates, the susceptibility of different host tissues, and the relative resistance of individual host genotypes. The excised-leaf inoculation technique appears to be especially useful for identifying the most resistant germ plasm for use in a breeding program because numerous repeated assays can be made to verify and compare responses of individual genotypes with minimal time and effort. This approach enables greatly increased efficiency of screening efforts because extensive vegetative cloning of potentially desirable genotypes is not necessary for repeated testing. In addition to its intended use for screening and selection, this technique also may be useful for evaluating influences of environmental factors on virulence of pathogens, susceptibility of host tissues, and expression of quantitative resistance. For example, influences of fertility treatments such as levels of N, P, and K on the susceptibility of host tissues, as previously determined or inferred from studies and observations in the field $(5,21)$, might be more precisely quantified by inoculating excised leaves of plants grown under different fertility regimes.

In each of two experiments, severity of symptoms across isolates increased in leaf tissues from positions 1 to 3 on stems, which represented the youngest and oldest leaves assayed, respectively (Tables 2 and 3). These relationships of symptom severity to leaf position correspond to results of inoculation experiments with spores of dematiaceous hyphomycetes applied to foliage of bermudagrass, in which symptoms are always more severe in lower, older leaves than in upper, younger leaves. The youngest, newly emerged leaves near stem tips often remain completely asymptomatic even when inoculated with spores of the most virulent isolates at high concentrations (14). This increased susceptibility of bermudagrass leaves to dematiaceous hyphomycetes with age, as observed both in excised leaves inoculated with mycelium and intact leaves inoculated with spores, corresponds to patterns of disease development in the field, where symptoms of stunting, dieback, and death of stands appear and intensify primarily after midseason as plant tissues mature $(5,14)$. Similarly, diseases incited by Curvularia spp. have been described as "senectotrophic" because they cause severe damage primarily in

TABLE 2. Mean lengths of necrosis observed for 26 fungal isolates plus controls inoculated onto excised leaves of bermudagrass from three positions on growing stems in experiment $1^{\mathrm{x}}$

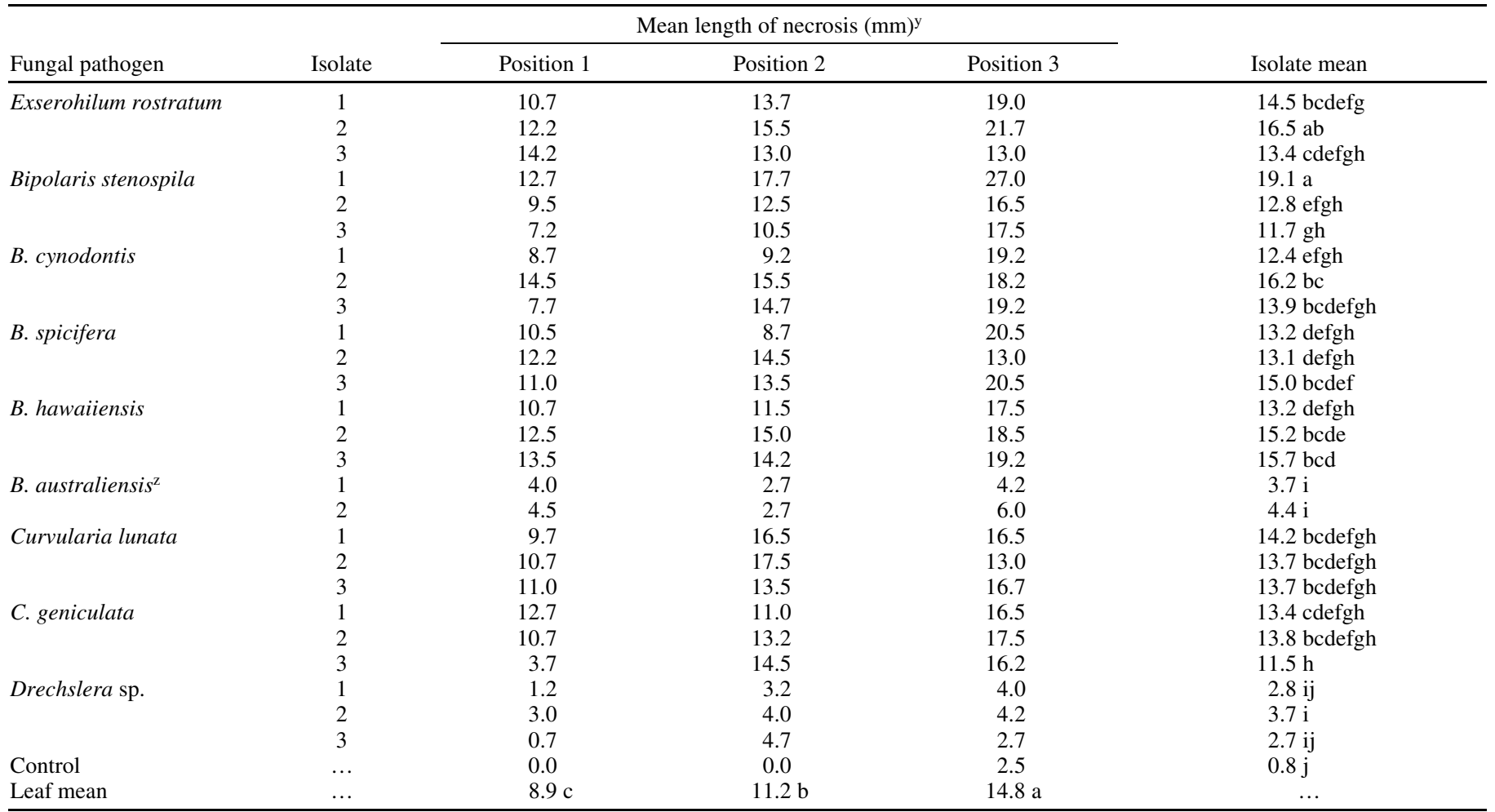

${ }^{x}$ Mean length of necrosis induced in leaves from different positions on stems. Means not followed by the same letter within columns or across rows differ significantly at $P=0.05$ according to Fisher's protected least significant difference (LSD) test. LSD for leaf position within isolate and pathogen isolate within leaf position $=4.8$.

y Position 1 = first fully formed and expanded leaf proximal from growing stem tip, position $2=$ second leaf, and position $3=$ third leaf.

${ }^{\mathrm{z}}$ Tentative identification. 
aged and senescent host tissues (5). The increased susceptibility of bermudagrass leaves to dematiaceous hyphomycetes with increasing age also is similar to the pattern of pathogenesis described for B. sorokiniana (Sacc.) Shoemaker on leaves of Poa pratensis L. In this pathosystem, severity of leafspot increases progressively on leaves that are sequentially older from tips of shoots, and severity is further increased by environmental and physiological factors such as light conditions (12) and herbicides (9) that promote senescence of leaves. In contrast, the increased susceptibility of bermudagrass leaves to dematiaceous hyphomycetes with increasing age is exactly opposite to the situation for Sclerotinia spp. on alfalfa, where the youngest leaves are most susceptible to pathogenesis, resistance increases in older leaves, and young stands are more severely damaged by disease than are older stands (13).

Numerous significant differences were observed between pathogen isolates in the extent of necrosis induced in excised bermudagrass leaves (Tables 2 and 3). However, variability among isolates within species was high, and few species-level differences were observed apart from low levels of necrosis caused by the slowgrowing isolates of $B$. australiensis (tentative identification) and Drechslera sp.

Some variability in results may have been caused by collection of leaves from stems selected at random from among 200 bermudagrass genotypes that undoubtedly differed in quantitative resistance to the dematiaceous hyphomycetous pathogens. However, such host genotypic differences would have been randomly distributed among all fungal species and isolates, so it is doubtful that these would have strongly influenced results apart from increasing experimental error.

Among isolates of seven relatively fast-growing species of fungal pathogens, the lack of consistent species-level differences in virulence with mycelial inoculation of excised leaves (Tables 2 and 3) was surprising because previous inoculations of foliage with spores at similar concentrations had consistently revealed numerous species-level differences in virulence $(14,15)$. In general, species with the largest conidia, such as B. stenospila and $E$. rostratum, always induced more severe foliar symptoms than species with smaller conidia, such as $B$. hawaiiensis and $B$. spicifera, and $C$. geniculata always induced the least severe symptoms with spore inoculations of foliage (14). With mycelial inoculation of excised leaves, however, these species-level differences in virulence were not apparent, and isolates of $C$. geniculata induced necrosis as extensive as most isolates of other species. It is suggested from these contrasting results that virulence differences observed with spore inoculations $(14,15)$ may result from differences in inoculum potential or infectiousness of spores of different sizes, even when similar numbers of germ tubes are produced from spores. With mycelial inoculation of excised leaves, any existing differences in spore inoculum potential or infectiousness (8) are circumvented, and few virulence differences then are apparent between species of dematiaceous hyphomycetes. Mycelial inoculation of excised leaves, therefore, may provide a more accurate assessment of the true virulence of pathogens with comparable growth rates in infected host tissue than do foliar inoculations with spores. Testing of this hypothesis might require simultaneous inoculations of leaf tissues with both mycelium and conidia of different isolates and species of dematiaceous hyphomycetes and evaluation of inoculum-isolate or inoculum-species interactions. Such direct comparisons between mycelial and conidial inoculum were not attempted in this study.

Apart from evaluating virulence of pathogens, the most valuable use of the excised-leaf inoculation technique may be to compare relative resistance and susceptibility of individual genotypes of bermudagrass to dematiaceous hyphomycetes. In this study, when 40 randomly selected genotypes were compared for re-

TABLE 3. Mean lengths of necrosis observed for 26 fungal isolates plus controls inoculated onto excised leaves of bermudagrass from three positions on growing stems in experiment $2^{\mathrm{x}}$

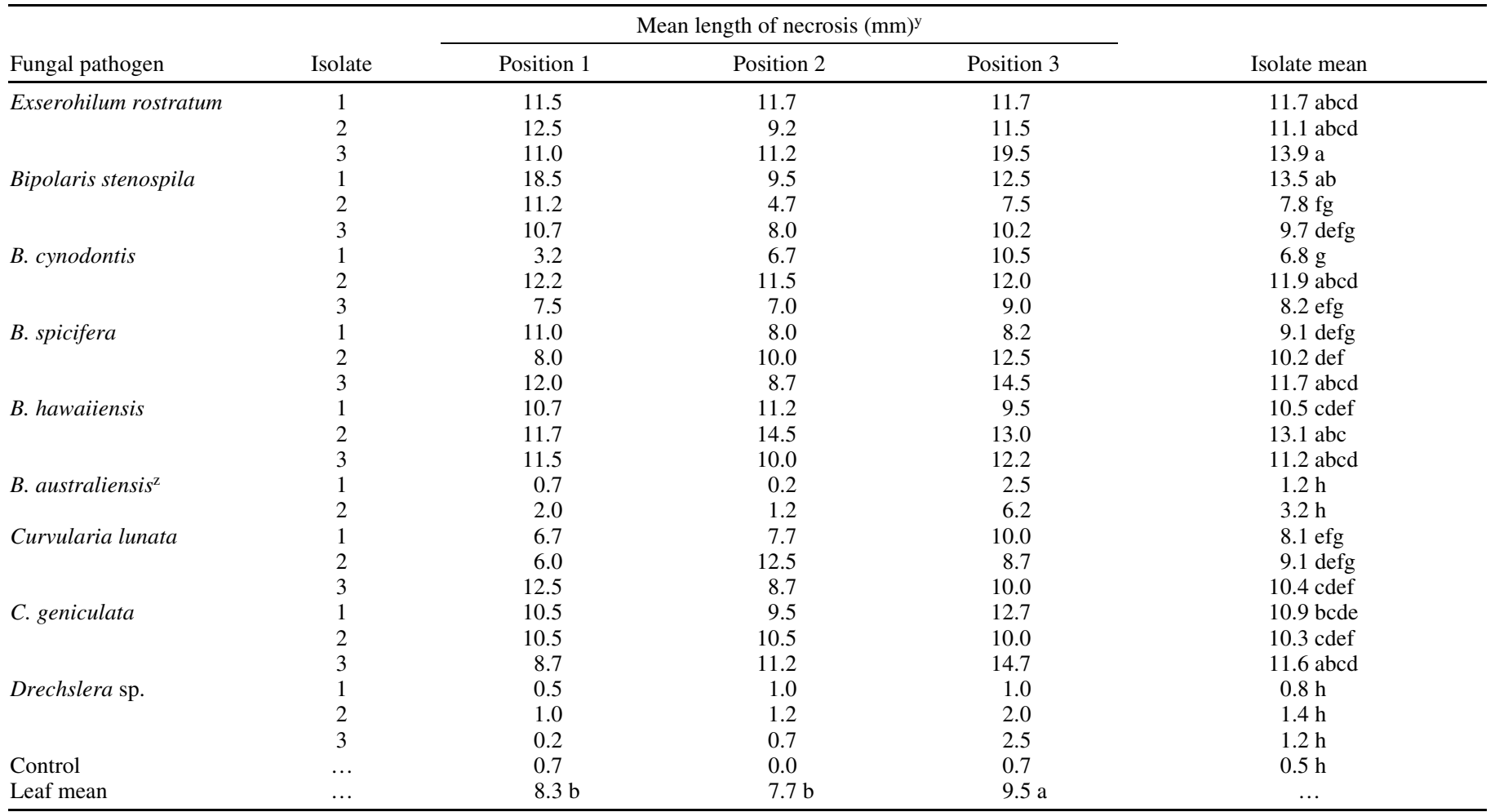

${ }^{x}$ Mean length of necrosis induced in leaves from different positions on stems. Means not followed by the same letter within columns or across rows differ significantly at $P=0.05$ according to Fisher's protected least significant difference (LSD) test. LSD for leaf position within isolate and pathogen isolate within leaf position $=5.0$.

y Position 1 = first fully formed and expanded leaf proximal from growing stem tip, position $2=$ second leaf, and position $3=$ third leaf.

${ }^{\mathrm{z}}$ Tentative identification. 
sponses to an isolate of E. rostratum, significant differences between genotypes were observed across experiments. The most relatively resistant genotypes developed only approximately half the extent of necrosis that occurred in the most susceptible (Fig. 1). The observed differences in host response represented a continuous numerical gradient between the susceptible and resistant extremes, and the occurrence of this gradient with an absence of

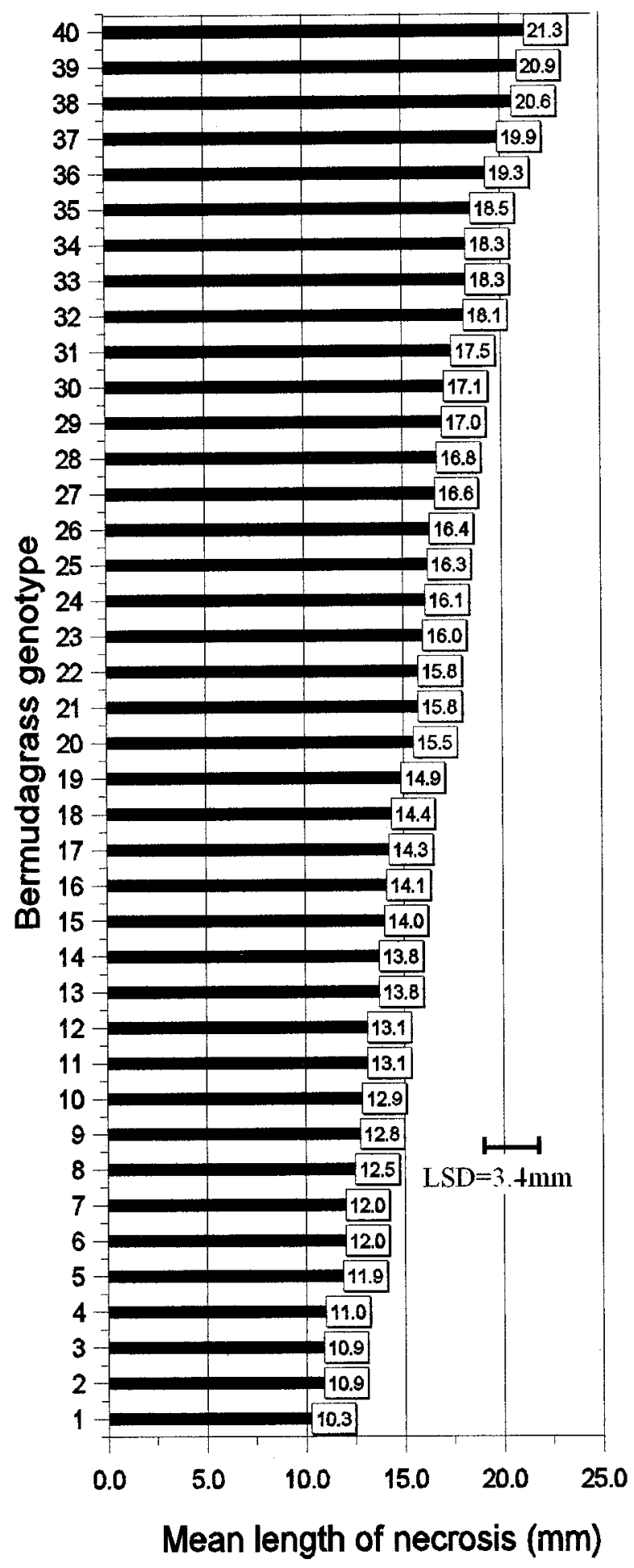

Fig. 1. Mean lengths of necrosis induced by an isolate of Exserohilum rostratum in excised leaves of 40 randomly selected bermudagrass genotypes. Means are for four replicate leaves from position 2 in each of two combined experiments. Least significant difference (LSD) is at $P=0.05$ according to Fisher's protected LSD test. Data represent mean lengths of continuous necrosis that extended proximally from the inoculated, cut tip of each leaf to the most distal, healthy-appearing, green tissue. discrete categories of phenotype clearly indicates that a quantitative form of resistance is represented by these genotypes $(2,3,7)$. Therefore, although direct mycelial inoculation of excised leaf tissues on wound sites may appear to represent a harsh inoculation method for fungi that are believed to infect plants primarily by germinated conidia $(8,9,12)$, the fact that differential host responses occurred consistently across experiments indicates that host resistance was effectively challenged but not overwhelmed in this system.

Differences in resistance to $E$. rostratum observed in excised leaves were verified by spore inoculations, in which less severe symptoms were induced in the most resistant genotypes than in the most susceptible (Table 4). However, differences in severity of symptoms between genotypes, as manifested by percentages of foliage in which symptoms were observed, were much more narrow with spore inoculations of total foliage than in excised leaves. This may occur if differential host responses to spore inoculations are expressed in only a small proportion of total foliage that may be approximately equivalent in susceptibility to leaves from position 2 on stems. Younger leaves of all genotypes may give similar resistant-appearing responses with little differential symptom development, and older leaves may give similar susceptible-appearing responses with strong symptom development. The excised leaf inoculation technique, therefore, may enable more precise differentiation between relative resistance of host genotypes if only tissues in which the best differential responses occur (likely leaves of position 2) are included in the test.

A logical use of the excised-leaf inoculation technique in screening bermudagrass for quantitative resistance might be as a secondary screening procedure following spore inoculations. Large numbers of plants might be mass-inoculated by spraying spores onto foliage as a primary screening procedure, and excised leaves of the most resistant-appearing plants might be inoculated subsequently with mycelium to more critically evaluate and compare host responses. The fact that significant differences in resistance were observed among only 40 randomly selected genotypes of unselected, common bermudagrass in this study indicates that genes for quantitative resistance might be selected even from relatively small populations. Additional potential uses of the excised-leaf inoculation technique include comparing crosseffectiveness of quantitative resistance to different genera and species of pathogens (15), determining the heritability of resistance phenotypes among appropriate genetic populations, and determining relationships of senescence or the physiological status of leaf tissues $(9,12)$ to the manifestation of genetic resistance and susceptibility.

TABLE 4. Severity of foliar symptoms following spore inoculations with Exserohilum rostratum in seven genotypes of bermudagrass selected for quantitative resistance (R) and susceptibility (S) by excised leaf inoculations

\begin{tabular}{lccc}
\hline & & \multicolumn{2}{c}{ Foliage with symptoms $(\%)^{\mathrm{x}}$} \\
\cline { 3 - 4 } Genotype $^{\mathrm{y}}$ & Response $^{\mathrm{z}}$ & 3 days & 10 days \\
\hline 40 & $\mathrm{~S}$ & $75 \mathrm{a}$ & $42 \mathrm{a}$ \\
38 & $\mathrm{~S}$ & $66 \mathrm{ab}$ & $40 \mathrm{a}$ \\
37 & $\mathrm{~S}$ & $70 \mathrm{bc}$ & $39 \mathrm{a}$ \\
39 & $\mathrm{~S}$ & $62 \mathrm{~cd}$ & $37 \mathrm{ab}$ \\
1 & $\mathrm{R}$ & $58 \mathrm{~d}$ & $33 \mathrm{bc}$ \\
2 & $\mathrm{R}$ & $56 \mathrm{~d}$ & $30 \mathrm{c}$ \\
3 & $\mathrm{R}$ & $55 \mathrm{~d}$ & $29 \mathrm{c}$ \\
\hline
\end{tabular}

${ }^{\mathrm{x}}$ Data are mean percentages of foliage estimated to manifest symptoms of pathogenesis (chlorosis and necrosis) at 3 and 10 days after spray inoculation with spores of E. rostratum. Means are based on eight replicate pots from two combined experiments. Means not followed by the same letter differ significantly at $P=0.05$ according to Fisher's protected least significant difference test.

${ }^{\mathrm{y}}$ Genotype numbers as listed in Figure 1.

${ }^{\mathrm{z}}$ Relative host response as determined by excised leaf inoculations. 


\section{ACKNOWLEDGMENTS}

I thank J. T. Davis, J. T. Flynn, J. D. Perry, and S. E. Wallace for technical assistance in all phases of this study.

\section{LITERATURE CITED}

1. Aung, M., Rowe, D. E., and Pratt, R. G. 1994. Necessity of replicated measurements for selection of alfalfa plants resistant or susceptible to stem inoculation by Sclerotinia trifoliorum. Plant Dis. 78:14-17.

2. Bernardo, R. 2002. Breeding for Quantitative Traits in Plants. Stemma Press, Woodbury, MN.

3. Birch, R. G. 2001. Disease resistance. Pages 356-363 in: Encyclopedia of Plant Pathology, Vol. II. O. C. Maloy and T. D. Murray, eds. John Wiley \& Sons, NY.

4. Burton, G. W., and Hanna, W. W. 1985. Bermudagrass. Pages 247-254 in: Forages The Science of Grassland Agriculture, 4th ed. M. E. Heath, R. L. Barnes, and D. S. Metcalfe, eds. Iowa State University Press, Ames.

5. Couch, H. B. 1995. Diseases of Turfgrasses. Krieger Publishing Co., Malabar, FL

6. Couch, H. B. 2000. The Turfgrass Disease Handbook. Krieger Publishing Co., Malabar, FL.

7. Geiger, H. W., and Heun, M. 1989. Genetics of quantitative resistance to fungal diseases. Annu. Rev. Phytopathol. 27:317-341.

8. Hodges, C. F. 1972. Influence of culture age and temperature on germination of Helminthosporium sorokinianum conidia and pathogenicity to Poa pratensis. Phytopathology 62:1133-1137.

9. Hodges, C. F. 1980. Interaction of sequential leaf senescence of Poa pratensis and pathogenesis by Drechslera sorokiniana as influenced by postemergence herbicides. Phytopathology 70:628-630.

10. Leonard, K. 1989. Proposed nomenclature for pathogenic races of Exserohilum turcicum on corn. Plant Dis. 73:776-777.
11. McCartney, C. A., Brule-Babel, A. L., and Lamari, L. 2002. Inheritance of race-specific resistance to Mycosphaerella graminicola in wheat. Phytopathology 92:138-144.

12. Nilson, K. M., Hodges, C. F., and Madsen, J. P. 1979. Pathogenesis of Drechslera sorokiniana leaf spot on progressively older leaves of Poa pratensis as influenced by photoperiod and light quality. Physiol. Plant Pathol. 15:171-176.

13. Pratt, R. G. 1995. Screening for resistance to Sclerotinia trifoliorum in alfalfa by inoculation of excised leaf tissue. Phytopathology 85:923-928.

14. Pratt, R. G. 2000. Diseases caused by dematiaceous fungal pathogens as potential limiting factors for production of bermudagrass on swine effluent application sites. Agron. J. 92:512-517.

15. Pratt, R. G. 2001. Occurrence and virulence of Bipolaris hawaiiensis on bermudagrass (Cynodon dactylon) on poultry waste application sites in Mississippi. Plant Dis. 85:1206.

16. Pratt, R. G., and Rowe, D. E. 2002. Enhanced resistance to Sclerotium rolfsii in populations of alfalfa selected for quantitative resistance to Sclerotinia trifoliorum. Phytopathology 92:204-209.

17. Shew, B. B., and Hollowell, J. E. 2000. Isolate aggressiveness and resistance to Sclerotinia minor on detached peanut leaves. (Abstr.) Phytopathology 90(suppl.):S72.

18. Sivanesan, A. 1987. Graminicolous species of Bipolaris, Curvularia, Drechslera, Exserohilum and their teleomorphs. Mycol. Pap. No. 158. CAB International Mycological Institute, Kew, Surrey, England.

19. Smiley, R. W., Dernoeden, R. H., and Clarke, B. B. 1992. Compendium of Turfgrass Diseases. The American Phytopathological Society, St. Paul, MN.

20. Steele, R. G. D., and Torrie, J. H. 1960. Principles and Procedures of Statistics. McGraw-Hill Book Co., New York.

21. Vargas, J. M., Jr. 1994. Management of Turfgrass Diseases. CRC Press, Boca Raton, FL

22. Wei, J.-K., Liu, K.-M., Chen, J.-P., Luo, P.-C., and Lee-Stadlemann, O. Y. 1988. Pathological and physiological identification of race $\mathrm{C}$ of Bipolaris maydis in China. Phytopathology 78:550-554. 